\begin{tabular}{lc} 
Sharif University of Technology & Scientia Iranica \\
SCIENTIA & Transactions F: Nanotechnology \\
I RAN ICA & http://scientiairanica.sharif.edu \\
\hline
\end{tabular}

\title{
Importance of activation energy and heat source on nanoliquid flow with gyrotactic microorganisms
}

\author{
T. Hayat ${ }^{\mathrm{a}, \mathrm{b}}$, I. Ullah ${ }^{\mathrm{a}, *}$, A. Alsaedi ${ }^{\mathrm{b}}$, and S. Asghar \\ a. Department of Mathematics, Quaid-I-Azam University 45320, Islamabad 44000, Pakistan. \\ b. Nonlinear Analysis and Applied Mathematics (NAAM) Research Group, Department of Mathematics, Faculty of Science, King \\ Abdulaziz University, P.O. Box 80203, Jeddah 21589, Saudi Arabia. \\ c. Department of Mathematics, CUT, Chak Shahzad, Park Road, Islamabad, Pakistan.
}

Received 6 February 2019; received in revised form 26 May 2019; accepted 21 September 2020

\section{KEYWORDS}

ESHS;

Activation energy;

Nanomaterials;

Power-law stretching;

Gyrotactic

microorganisms.

\begin{abstract}
This article addresses salient features of gyrotactic microorganisms and activation energy in the flow of nanofluid by rotating disks. An ESHS process is implemented to examine the thermal transport characteristics. Additionally, the nanoparticle mass flux condition is considered and the solutions are numerically computed. Impacts of various physical variables appearing in the solutions of non-linear systems are carefully analyzed. The current work identifies that temperature distribution of nanoliquid enhances via thermophoresis and Brownian diffusion variables. Moreover, activation energy and temperature difference parameters diminish nanoparticle concentration. Comparative study is provided in order to validate the outcomes.
\end{abstract}

(C) 2020 Sharif University of Technology. All rights reserved.

\section{Introduction}

Analysis of nanofluids has received special attention in recent research. Such interest is stimulated by the fact that nanotechnology is now considered a noteworthy factor that influences the modern insurgency of the present century. In the previous couple of years, numerous scientists have been concentrating on demonstrating thermal conductivity and looking at the changed viscosities of nano-liquids. There is no doubt that heat transfer liquids like water and minerals, ethylene glycol and oil play important roles in many industrial sites, including chemical production, air conditioning, power generation, microelectronics, transportation, food, pharmaceuticals, etc. It is also recognized fact that most heat transfer liquids, for example, motor oil,

*. Corresponding author. Tel.: +92-5190642172 E-mail address: ikramullah@math.qau.edu.pk (I. Ullah)

doi: $10.24200 /$ sci. 2020.52851 .2927 ethylene glycol and water, have constrained/poor capabilities in terms of thermal features which thus create limitations in thermal procedures. On the other hand, metals are considered to have a thermal performance three times higher than these liquids. Various studies have been undertaken for improvement of the thermal conductivity of such conventional heat transfer liquids. A creative technique for raising the heat exchange of base liquids is to drop small particles into it [1]. Commonly, the nanoparticles are compacts of oxides (titania, alumina and carbides, copper oxide,) and metals including (gold and copper). Carbon nanotubes and diamond are also used in nano-liquids. The base liquids include ethylene glycol, oil, water, biofluids, some lubricants and polymer solutions. The scope of nanoliquid can be noted in many biomedical and engineering systems, such as computers, nuclear reactors, car engines, cancer therapy, solar energy, radiators, safer surgeries, safety issues emerging in nuclear reactors and X-rays. Moreover, nanoliquids can be used as a cooling agent in micro machines in micro reactors, 
cars, airplanes, CPU etc. The most important characteristics of such liquids are better spreading, sufficient viscosity, stability, dispersion and wetting on solid surfaces [2]. The addition of nanoparticles may result in a coefficient of heat transportation diminution but literature reveals the improvement in heat transport. Numerous mechanisms for illustration of thermal diffusion, micro-convection, thermophoretics, Brownian movement, interface of nanoparticles, and particle to particle coupling have been elaborated to illustrate the improvement regarding heat transport. Some remarkable endeavors in this area can be seen by refs. [3-35].

A reaction that releases energy initially needs some quantity of energy to achieve its energy releasing levels. This initial input energy, which precedes the reaction, is referred to as activation energy. Activation energy was introduced by Svante Arrhenius. Such energy has a noteworthy association in regard to assessment of a reaction [36]. Geothermal reservoirs, thermal oil recovery, chemical engineering, nuclear reactor chilling etc. are some examples of combined chemical reaction and activation energy. Initially, Bestman [37] addressed the simultaneous aspect of activation energy, along with chemical reaction, for convective mass transport analysis. Awad et al. [38] addressed unsteady stretched flow in a rotating liquid with activation energy and chemical reactions. Features of activation energy and chemical reactions in unsteady convective flow bounded by a radiated surface are scrutinized by Makinde et al. [39], who discussed the features of activation energy in radiated flow. Mixed convection radiated flow accounting for activation energy is studied by Maleque [40]. The flow of Maxwell materials via a surface subject to activation energy is explored by Mustafa et al. [41]. Abbas et al. [42] studied the impact of activation energy in chemically reacted Casson liquid flow. Recently, Zeeshan et al. [43] and Archana and Mahanthesh [44] numerically explored the behavior of activation energy in flow by considering Casson liquid and viscous models.

The objective of this paper is to analyze bioconvection flow comprised of both microorganisms and nanoparticles. A rotating disk with power-law stretching is used. A Buongiorno model has been implemented and zero mass flux condition is imposed. Further combined aspects of exponential heat source, binary chemical reactions and activation energy are addressed. The governing expressions are reduced to a set of nonlinear boundary layer problems. The numerical study is performed using the NDSolve technique $[45,46]$ and the behavior of active variables are sketched via graphs. Tables are presented to reflect the computational analysis of skin frictions and Nusselt number. Moreover, a comparative table is also shown to justify the current outcomes with previous analyses. Major outcomes are listed in the conclusions.

\section{Mathematical development}

The flow, temperature, microorganism and concentration distributions are governed by the continuity expression, momentum expression, energy and mass transport expressions with exponential space dependent internal heat source and activation energy (see Figure 1). The following assumptions are made for the governing problem.

(i) Viscous nanomaterials are used;

(ii) Flow is steady, incompressible and two dimensional;

(iii) The nanoparticles have uniform size and shape;

(iv) There is relative movement between nanoparticles and regular liquid;

(v) Nanoparticles have no impact on the velocity of microorganisms and swimming direction;

(vi) The suspended nanoparticles are stable and do not accumulate in the liquid;

(vii) Thermophoretic and Brownian diffusions are present.

The problem statement using the above assumptions leads to the following equations [47-50]:

$$
\begin{aligned}
& \frac{\partial(r u)}{\partial r}+\frac{\partial(r w)}{\partial z}=0, \\
& u \frac{\partial u}{\partial r}+w \frac{\partial u}{\partial z}-\frac{v^{2}}{r}=\nu \frac{\partial^{2} u}{\partial z^{2}}, \\
& u \frac{\partial v}{\partial r}+w \frac{\partial v}{\partial z}+\frac{u v}{r}=\nu \frac{\partial^{2} v}{\partial z^{2}},
\end{aligned}
$$

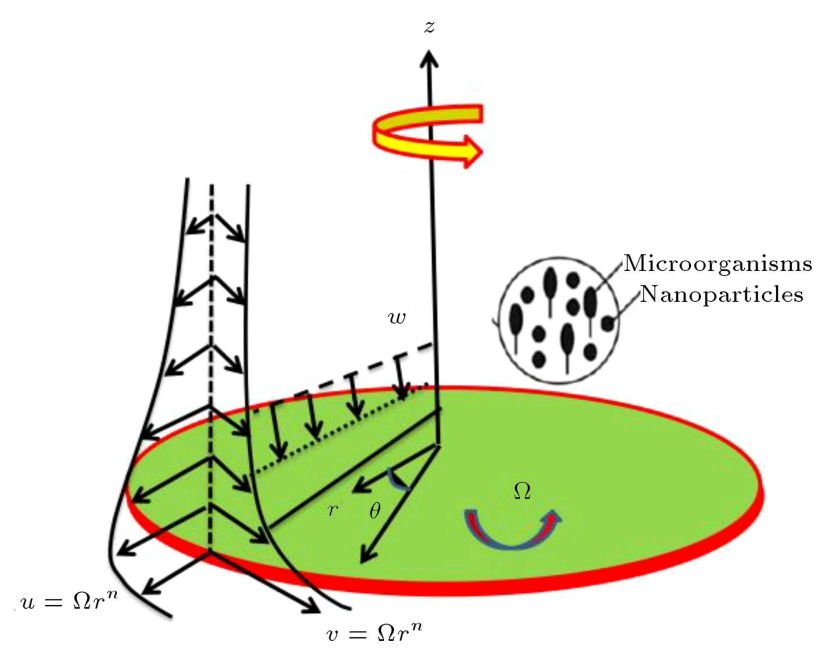

Figure 1. Physical sketch. 


$$
\begin{aligned}
& u \frac{\partial T}{\partial r}+w \frac{\partial T}{\partial z}=\alpha_{f} \frac{\partial^{2} T}{\partial z^{2}}+\tau\left(D_{B} \frac{\partial C}{\partial z} \frac{\partial T}{\partial z}+\frac{D_{T}}{T_{\infty}}\left(\frac{\partial T}{\partial z}\right)^{2}\right) \\
& +\frac{Q_{0}\left(T-T_{\infty}\right)}{\left(\rho c_{p}\right)_{f}} e^{-z n_{1} \sqrt{\frac{\Omega}{\nu}} r \frac{n-1}{2}} \\
& u \frac{\partial C}{\partial r}+w \frac{\partial C}{\partial z}=D_{B}\left(\frac{\partial^{2} C}{\partial z^{2}}\right)+\frac{D_{T}}{T_{\infty}}\left(\frac{\partial^{2} T}{\partial z^{2}}\right) \\
& -k_{r}^{2}\left(C-C_{\infty}\right)\left(\frac{T}{T_{\infty}}\right)^{m} \exp \left(\frac{-E_{a}}{\kappa T}\right) \\
& u \frac{\partial N}{\partial r}+w \frac{\partial N}{\partial z}=-\frac{b W_{c}}{\Delta C}\left[N \frac{\partial^{2} C}{\partial z^{2}}+\frac{\partial C}{\partial z} \frac{\partial N}{\partial z}\right] \\
& +D_{n} \frac{\partial^{2} N}{\partial z^{2}} \\
& u=a r u_{w}(r)=a r^{n}, \quad v=\Omega r u_{w}(r)=\Omega r^{n}, \\
& w=0, \quad T=T_{w}, \quad D_{B} \frac{\partial C}{\partial z}+\frac{D_{T}}{T_{\infty}} \frac{\partial T}{\partial z}=0, \\
& N=N_{w} \quad \text { at } \quad z=0, \\
& u \rightarrow 0, \quad v \rightarrow 0, \quad T \rightarrow T_{\infty}, \quad C \rightarrow C_{\infty} \\
& N \rightarrow N_{\infty} \quad \text { as } \quad z \rightarrow \infty .
\end{aligned}
$$

Here, $(u, v, w)$ are the velocity components in $(r, \varphi, z)$; $\nu$ the kinematic viscosity, $\tau=\frac{(\rho c)_{p}}{(\rho c)_{f}}$ the heat capacity ratio; $n_{1}$ the exponential index; $\alpha_{f}$ the thermal diffusivity of nanoliquid; $\rho$ the liquid density; $Q_{0}$ heat generation/absorption variable; $E_{a}$ the nondimensional activation energy; $D_{B}$ the diffusion coefficient; $D_{T}$ the coefficient of thermophoretic diffusion; $b$ the chemotaxis constant; $\left(T, T_{w}, T_{\infty}\right)$ fluid, surface and ambient temperatures, fluid, surface and ambient concentrations are $\left(C, C_{w}, C_{\infty}\right)$; the concentrations of microorganisms are $\left(N, N_{w}, N_{\infty}\right) ; D_{n}$ the diffusivity of microorganisms; and $W_{c}$ the maximum cell swimming speed.

Note that energy expression is modeled via exponential heat source for the distribution of internal temperature [51,52]. Further, in Eq. (5) $\kappa=8.61 \times$ $10(\mathrm{eV} / \mathrm{K})$ as the Boltzmann constant, $k_{r}^{2}$ designates the reaction rate, and $m(-1<m>1)$ the fitted rate constant. Consider [16,47]:

$$
\begin{aligned}
& u=\Omega r^{n} f^{\prime}(\xi), \quad v=\Omega r^{n} g(\xi), \\
& w=-\sqrt{\Omega \nu} r^{\frac{n-1}{2}}\left(\frac{3+n}{2} f(\xi)+\frac{n-1}{2} \xi f^{\prime}(\xi)\right), \\
& \xi=\sqrt{\frac{\Omega}{\nu}} r^{\frac{n-1}{2}} z, \quad \theta(\xi)=\frac{T-T_{\infty}}{T_{w}-T_{\infty}}, \\
& \phi(\xi)=\frac{C-C_{\infty}}{C_{\infty}}, \quad S(\xi)=\frac{N}{N_{w}} .
\end{aligned}
$$

It is assumed that the longitudinal pressure gradient is zero. On using the above transformations, expression (1) is trivially verified and Eqs. (2)-(8) give:

$$
\begin{aligned}
& f^{\prime \prime \prime}-n f^{\prime 2}+\left(\frac{n+3}{2}\right) f f^{\prime \prime}+g^{2}=0 \\
& g^{\prime \prime}-(n+1) f^{\prime} g+\left(\frac{n+3}{2}\right) f g^{\prime}=0, \\
& \theta^{\prime \prime}+\operatorname{Pr}\left(\frac{n+3}{2}\right) f \theta^{\prime}+N_{b} \theta^{\prime} \phi^{\prime}+\operatorname{Pr} Q \exp \left(-n_{1} \xi\right) \\
& +N_{t} \theta^{\prime 2}=0 \\
& \phi^{\prime \prime}+\left(\frac{n+3}{2}\right) \operatorname{Le} f \phi^{\prime}+\frac{N_{t}}{N_{b}} \theta^{\prime \prime} \\
& -\operatorname{Le} \alpha_{1}(1+\delta \theta)^{m} \phi \exp \left(\frac{-E}{1+\delta \theta}\right)=0, \\
& S^{\prime \prime}+\left(\frac{n+3}{2}\right) S_{c} f S^{\prime}-\operatorname{Pe} \phi^{\prime \prime} S-\operatorname{Pe} \phi^{\prime} S=0 \\
& f^{\prime}(0)=\alpha, \quad f(0)=0, \quad g(0)=1, \\
& \theta(0)=1, \quad \phi^{\prime}(0)+\frac{N_{t}}{N_{b}} \theta^{\prime}(0)=0, \quad S(0)=1, \\
& f^{\prime}(\infty) \rightarrow 0, \quad g(\infty) \rightarrow 0, \quad \theta(\infty) \rightarrow 0, \\
& \phi(\infty) \rightarrow 0, \quad S(\infty) \rightarrow 0
\end{aligned}
$$

where prime designates differentiation via $\xi, N_{t}$ the thermophoresis parameter, Pe the bioconvection Peclet number, $N_{b}$ the Brownian motion variable, Le denotes Lewis number, Pr the Prandtl number, $Q$ the (ESHS) variable, Sc the Schmidt number, $\delta$ the temperature difference variable, $\alpha_{1}$ stands for non-dimensional reaction rate, $E$ the dimensionless activation energy and $\alpha$ the ratio of stretching rate to angular velocity. These quantities are defined by: 


$$
\begin{array}{lrl}
N_{t}=\frac{(\rho c)_{p}}{(\rho c)_{f}} \frac{D_{T}\left(T_{w}-T_{\infty}\right)}{T_{\infty} \nu}, & N_{b}=\frac{(\rho c)_{p}}{(\rho c)_{f}} \frac{D_{B} C_{\infty}}{\nu} \\
Q=\frac{Q_{0}}{\Omega\left(\rho c_{p}\right)_{f}}, & \alpha=\frac{a}{\Omega}, & \delta=\frac{\left(T_{w}-T_{\infty}\right)}{T_{\infty}}, \\
\mathrm{Le}=\frac{\nu}{D_{B}}, & \operatorname{Pr}=\frac{\nu}{\alpha_{f}}, & S_{c}=\frac{\nu}{D_{n}}, \\
\alpha_{1}=k_{r}^{2} / \Omega, & E=\frac{E_{a}}{\kappa T_{\infty}}, & \mathrm{Pe}=\frac{b W c}{D_{n}} .
\end{array}
$$

The typical quantities of key interest are the skin frictions in radial and azimuthal directions $\left(C_{f_{r}}, C_{f_{\phi}}\right)$, temperature gradient $\left(\mathrm{Nu}_{r}\right)$ and gradient of motile microorganisms $\left(N n_{r}\right)$.

$$
\begin{aligned}
& C_{f_{r}}=\frac{\mu\left(\frac{\partial u}{\partial z}+\frac{1}{r} \frac{\partial w}{\partial \phi}\right)}{\rho\left(r^{n} \Omega\right)^{2}}, \quad C_{f_{\phi}}=\frac{\mu\left(\frac{\partial v}{\partial z}+\frac{1}{r} \frac{\partial w}{\partial \phi}\right)}{\rho\left(r^{n} \Omega\right)^{2}} \\
& \mathrm{Nu}_{r}=\frac{-k r\left(\frac{\partial T}{\partial z}\right)_{z=0}}{k\left(T_{w}-T_{\infty}\right)} \\
& N n_{r}=\frac{-D_{n} r\left(\frac{\partial N}{\partial z}\right)_{z=0}}{D_{n} N_{w}}
\end{aligned}
$$

In dimensionless form, one has:

$$
\begin{aligned}
& \sqrt{\operatorname{Re}_{r}} C_{f_{r}}=f^{\prime \prime}(0), \quad \sqrt{\operatorname{Re}_{r}} C_{f_{\phi}}=g^{\prime}(0), \\
& \left(\operatorname{Re}_{r}\right)^{-0.5} \mathrm{Nu}_{r}=-\theta^{\prime}(0), \\
& \left(\operatorname{Re}_{r}\right)^{-0.5} \mathrm{Nn}_{r}=-S^{\prime}(0),
\end{aligned}
$$

where $\operatorname{Re}_{r}=u_{w} r / \nu$ depicts local Reynolds number.

\section{Computational scheme}

The governing problem consists of a non-linear system. Therefore, it is not possible to find exact solutions. However, an approximate solution can be computed through various techniques such as analytical and numerical etc. Here, in the problem considered, the NDSolve based Shooting scheme is employed. This technique is a numerical solver of differential systems. With the aid of NDSolve one can tackle different ODEs systems as well as special PDEs systems. General ODEs systems possess a number of equations $n$ (i.e., $q_{1}, q_{2}, q_{3} \cdots q_{n}$ ), independent variable $x$, number of dependent variables $n$ (i.e., $v_{1}, v_{2}, v_{3} \cdots v_{n}$ ), and boundary conditions according to the order of the PDEs system. Using the NDSolve technique, this system can be computed as follows:

NDSolve $\left[\left\{q_{1}, q_{2}, q_{3} \cdots q_{n}\right.\right.$, boundary conditions $\}$,

$$
\left\{v_{1}, v_{2}, v_{3} \cdots v_{n}\right\},\left\{x, x_{\min }, x_{\max }\right\} .
$$

This technique attains exceptional accuracy and is stable unconditionally. Furthermore, it provides the best outcomes in minimum CPU time and avoids lengthy expressions.

\section{Discussion}

The current intention is to predict the characteristics of sundry variables on velocity components (radial $f^{\prime}(\xi)$ and tangential $g(\xi)$ ), temperature $\theta(\xi)$, concentration $\phi(\xi)$, and nanoparticle motile density $S(\xi)$. The values used for involved variables are: $n=0.5, \operatorname{Pr}=0.7=$ $\mathrm{Pe}=S_{c}, \delta=0.3=N_{b}, N_{t}=0.1$, Le $=1.0=\alpha_{1}$, $m=0.5, Q=0.2=\alpha=n_{1}$, and $E=3.0$. Figure 2 presents the characteristics of $\alpha$ on $f^{\prime}(\xi)$. In fact, larger values of $\alpha$ result in an enhancement of stretching rate near the disk and so $f^{\prime}(\xi)$ increase. Figures 3 and 4 are interpreted to inspect the physical impacts of embedding variables $\alpha$ and $n$ on tangential velocity $g(\xi)$. Here, radial velocity enhances for higher $\alpha$ whereas a reverse trend is observed for power law index $n$ (see Figure 4). Variations of $\theta(\xi)$ for multiple values of $N_{t}$ are pointed out in Figure 5. Obviously, larger $N_{t}$ yield higher $\theta(\xi)$ and corresponding thermal layer thickness. Physically higher $N_{t}$ show stronger thermophoretic force on nanoparticles. Therefore, a large number of nanoparticles is shifted towards ambi-

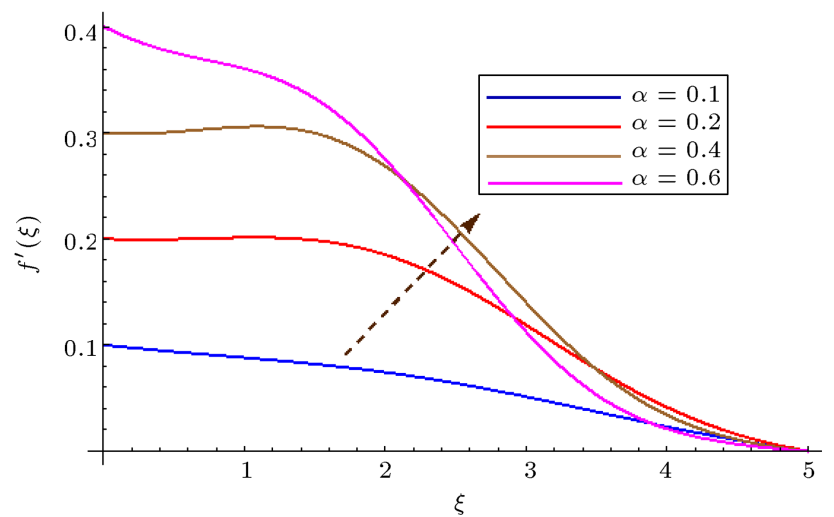

Figure 2. Behavior of $f^{\prime}$ via $\alpha$.

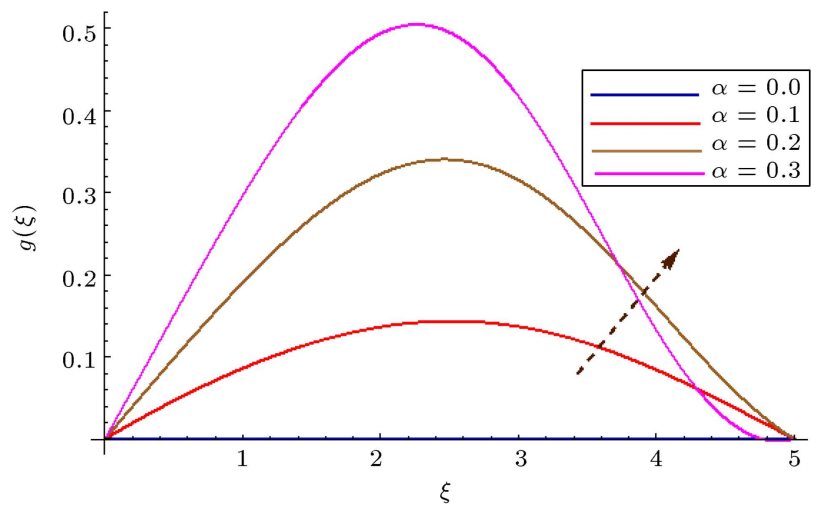

Figure 3. Behavior of $g$ via $\alpha$. 


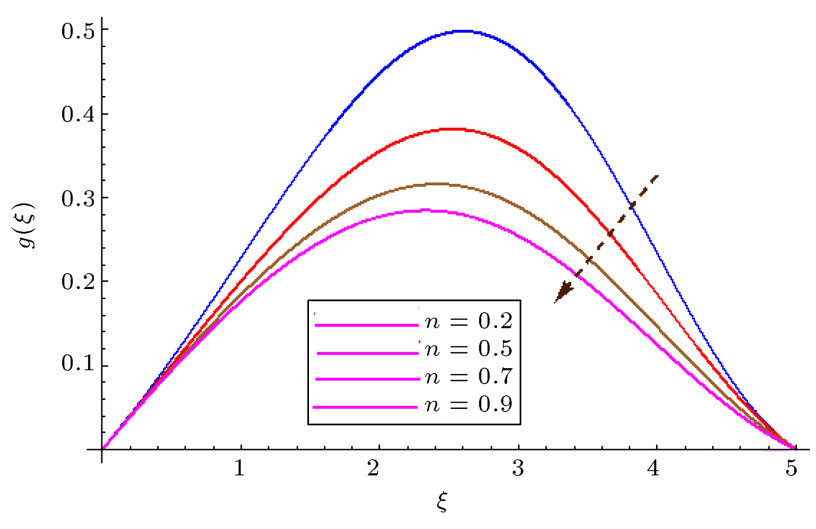

Figure 4. Behavior of $g$ via $n$.

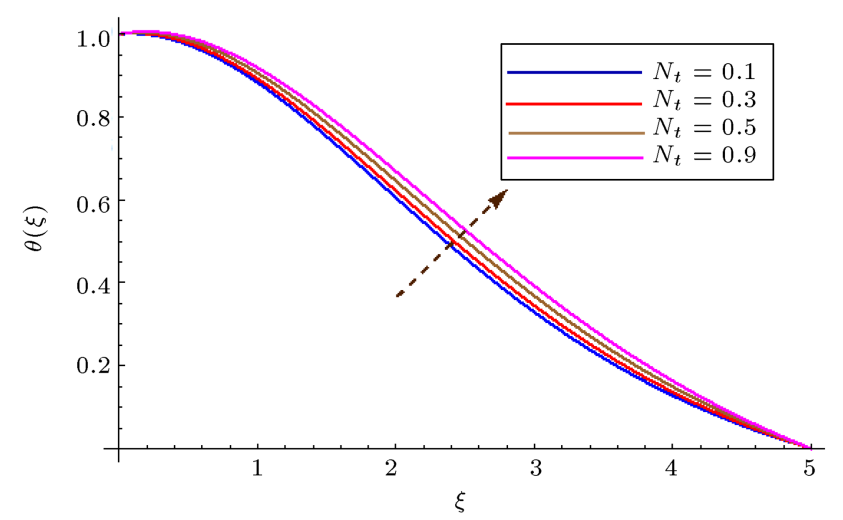

Figure 5. Behavior of $\theta$ via $N_{t}$.

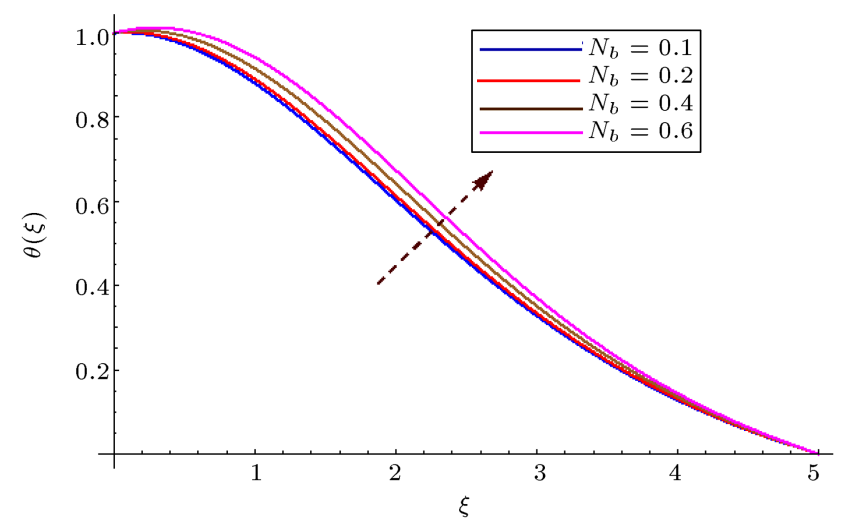

Figure 6. Behavior of $\theta$ via $N_{b}$.

ent liquid, which enhances the thermal field. Features of $N_{b}$ on $\theta(\xi)$ are depicted in Figure 6. Higher $N_{b}$ augments the material particles random motion, due to which, more heat is produced. That is why the $\theta(\xi)$ increases. Characteristic of ESHS parameter $Q$ on $\theta(\xi)$ is illustrated in Figure 7 . Here, the thermal field improves for larger $Q$. In view of physics, an increment in $Q$ supplies heat in the fluid and the thermal field rises. Figure 8 shows the changes in $N_{b}$ for $\phi(\xi)$. It has been pointed out that by enhancing $N_{b}$, an enhancement occurs in the thermal field. In fact, higher $N_{t}$ improves the nanoparticle movement rates, which

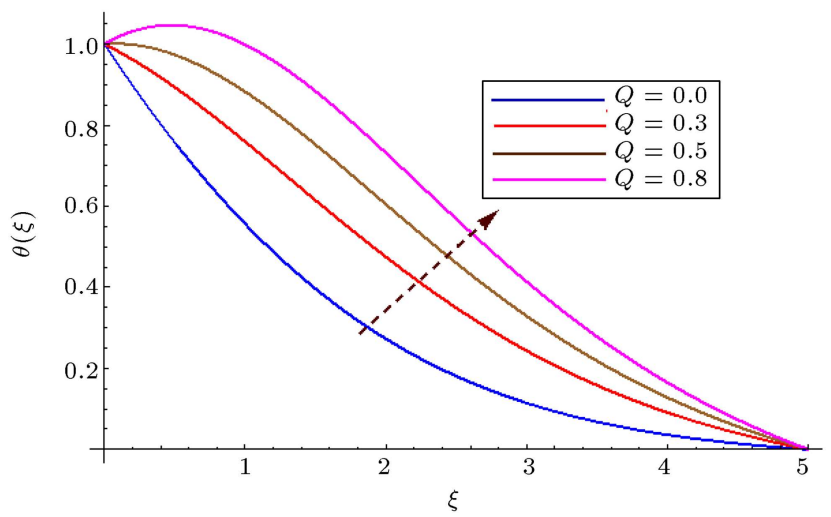

Figure 7. Impact of $\theta$ via $Q$.

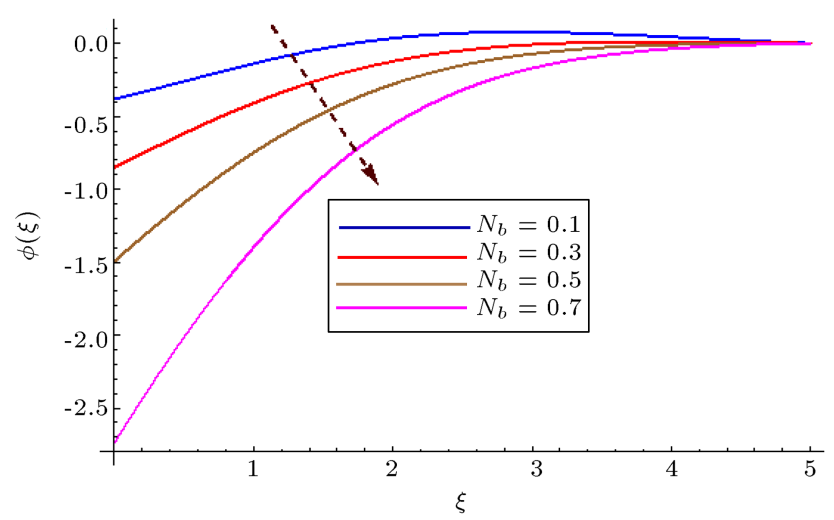

Figure 8. Behavior of $\phi$ through $N_{b}$.

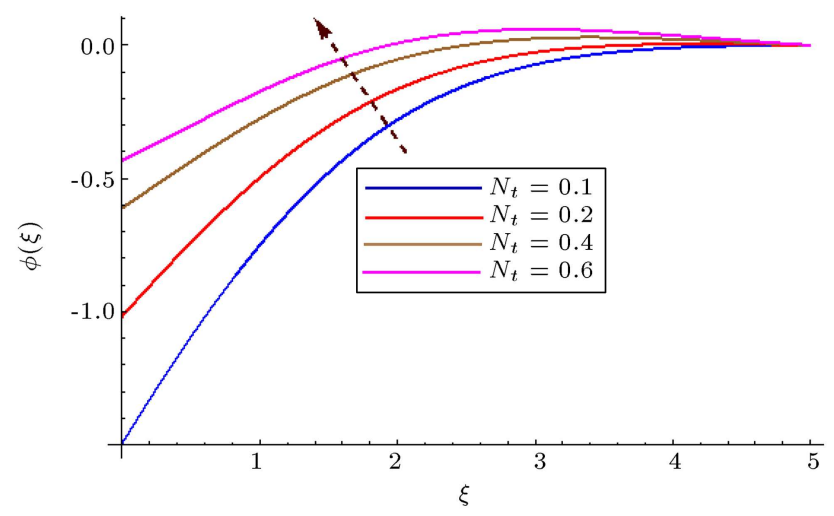

Figure 9. Behavior of $\phi$ through $N_{t}$.

have distinct velocities because of Brownian diffusion. Figure 9 illustrates effect of thermophoresis parameter $N_{t}$ on $\phi(\xi)$. The plot indicates that increasing estimations of $N_{t}$ augment concentration. In a physical sense, larger $N_{t}$ capitulate an increment in thermophoretic force and this frequently shifts nanoparticles from a higher to lower concentration region and so $\phi(\xi)$ boosts. Variations of $\phi(\xi)$ via $E$ are plotted in Figure 10. Here, $\phi(\xi)$ enhances for larger activation energy parameter $E$. Physically an increment in $E$ decays the exponential factor, which ultimately generates the chemical reaction due to which $\phi(\xi)$ enhances. Enhancement 


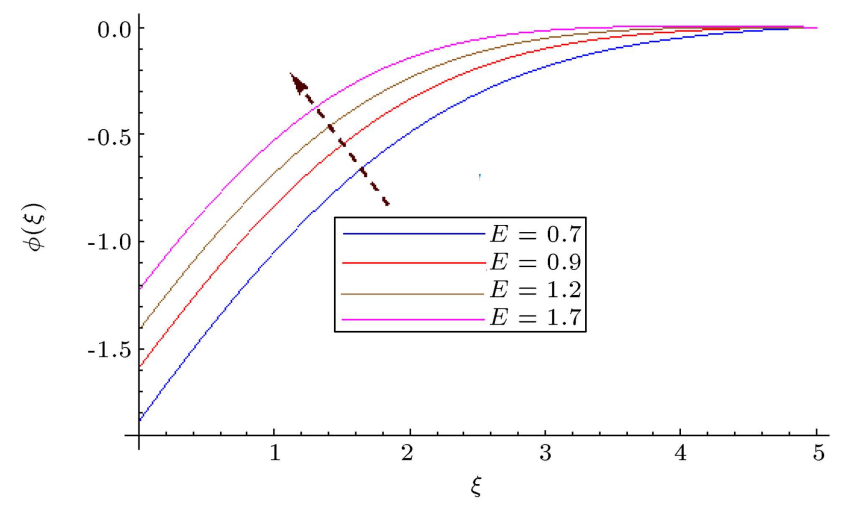

Figure 10. Behavior of $\phi$ through $E$.

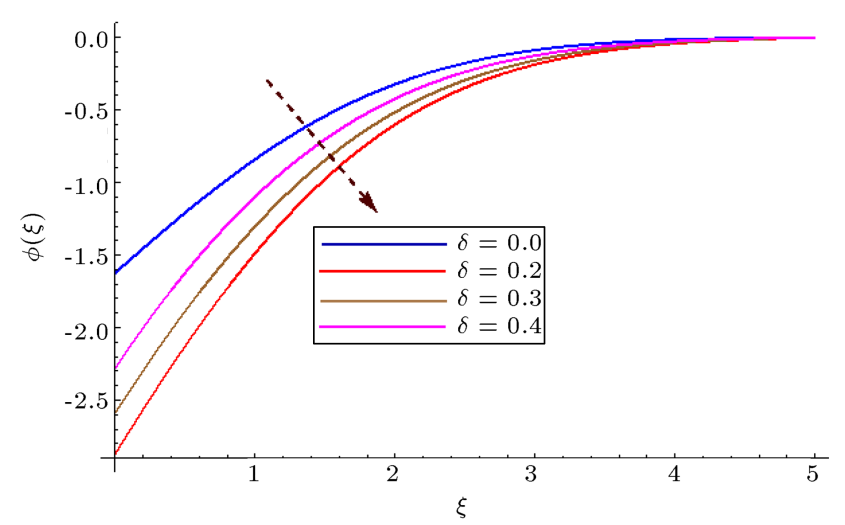

Figure 11. Behavior of $\phi$ through $\delta$.

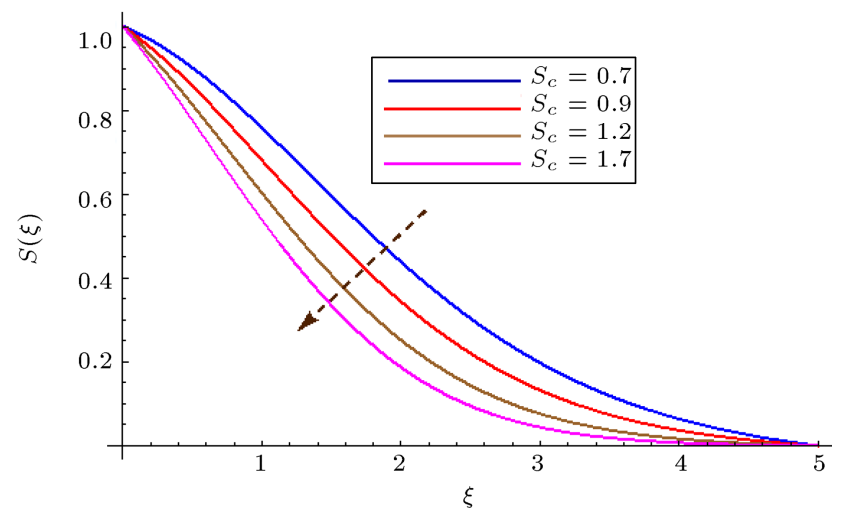

Figure 12. Effect of $S$ through $S_{c}$.

in $\delta$ leads to a decay in concentration distribution. This behavior is depicted in Figure 11. $S(\xi)$ depicts decaying behavior against $S_{c}$ (see Figure 12). The feature of $\mathrm{Pe}$ on $S(\xi)$ is exposed in Figure 13. It is found that $S(\xi)$ increases when $\mathrm{Pe}$ is enhanced. It is because larger Pe causes decay in the diffusivity of microorganisms and thus the motile density of liquid reduces. Aspects of diverse embedding variables on skin frictions, local Nusselt number and motile density number of microorganisms are reported in Tables 1 and 2. From Table 1 , it is noted that skin friction along radial direction $\left(\left(\operatorname{Re}_{r}\right)^{0.5} C_{f r}\right)$ enhances for larger

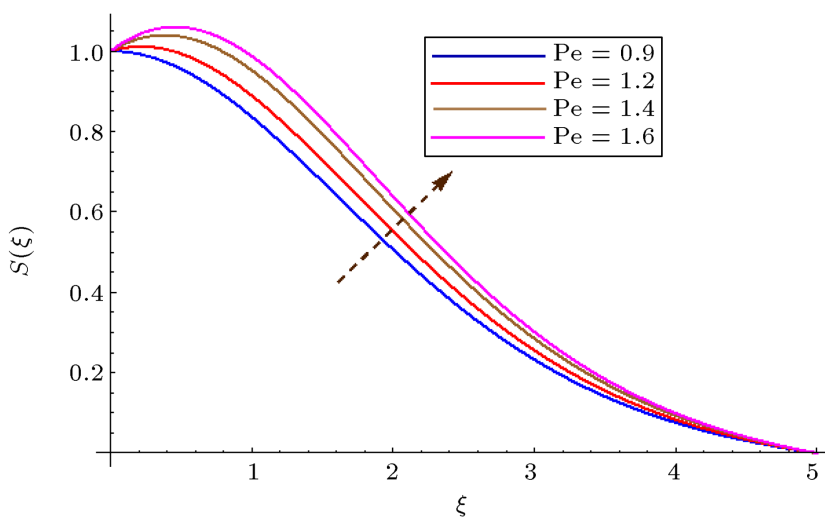

Figure 13. Effect of $S$ through Pe.

Table 1. Numerical estimations of skin frictions for distinct values of $n$ and $\alpha$ when $\operatorname{Pr}=0.7=\mathrm{Pe}=S_{c}$, $\delta=0.3=N_{b}, N_{t}=0.1$, Le $=1.0=\alpha_{1}, m=0.5$, $Q=0.2=n_{1}$, and $E=3.0$.

\begin{tabular}{cccc}
\hline $\boldsymbol{n}$ & $\boldsymbol{\alpha}$ & $\left(\mathrm{Re}_{\boldsymbol{r}}\right)^{\mathbf{0 . 5}} \boldsymbol{C}_{\boldsymbol{f} \boldsymbol{r}}$ & $\left(\mathrm{Re}_{\boldsymbol{r}}\right)^{\mathbf{0 . 5}} \boldsymbol{C}_{\boldsymbol{f} \boldsymbol{\varphi}}$ \\
\hline 0.5 & 0.2 & 0.0054969 & 0.1799612 \\
0.9 & & 0.0408849 & 0.0202541 \\
1.0 & & 0.0559298 & 0.0408849 \\
0.5 & 0.2 & 0.0054969 & 0.2000000 \\
& 0.5 & 0.1580423 & 0.2707312 \\
& 1.0 & 0.6800625 & 0.6800621 \\
\hline
\end{tabular}

Table 2. Numerical data of Nusselt number and density of the motile microorganisms for $n, N_{b}, N_{t}$ and $E$ when $\operatorname{Pr}=0.7=\operatorname{Pe}=S_{c}, \delta=0.3, \mathrm{Le}=1.0=\alpha_{1}$, $Q=0.2=\alpha=n_{1}$ and $m=0.5$.

\begin{tabular}{cccccc}
\hline $\boldsymbol{n}$ & $\boldsymbol{N}_{\boldsymbol{b}}$ & $\boldsymbol{N}_{\boldsymbol{t}}$ & $\boldsymbol{E}$ & $-\boldsymbol{\theta}^{\prime}(\mathbf{0})$ & $-\boldsymbol{S}^{\prime}(\mathbf{0})$ \\
\hline 0.2 & 0.3 & 0.1 & 3.0 & 0.152402 & 0.215945 \\
0.5 & & & & 0.027784 & 0.050162 \\
1.0 & & & & 0.047204 & 0.030000 \\
0.5 & 0.1 & 0.1 & 3.0 & 0.024494 & 0.050838 \\
& 0.3 & & & 0.027784 & 0.050162 \\
& 0.8 & & & 0.090303 & 0.043765 \\
0.5 & 0.1 & 0.1 & 3.0 & 0.027784 & 0.050162 \\
& & 0.4 & & 0.036857 & 0.054499 \\
& & 0.6 & & 0.050806 & 0.060307 \\
0.5 & 0.3 & 0.1 & 1.0 & 0.027686 & 0.050096 \\
& & & 2.0 & 0.027706 & 0.050139 \\
0.2 & 0.3 & 0.1 & 3.0 & 0.027784 & 0.050162 \\
\hline
\end{tabular}

estimations of power law index $n$, whereas skin friction along the azimuthal direction $\left(\left(\operatorname{Re}_{r}\right)^{0.5} C_{f \phi}\right)$ has a reverse behavior. From Table 2, it is pointed out that local Nusselt number $\left(\mathrm{Nu}_{r}\right)$ is enhanced for $N_{b}, N_{t}$ and $E$. This table also provides the information that rising values of $N_{b}$ and $E$ decay $S^{\prime}(0)$. Table 3 shows a comparison of the presented investigation with Chen 
Table 3. Comparative data of $f^{\prime \prime}(0)$ and $g^{\prime}(0)$ for various values of $n$ and $\alpha$ when $Q=\alpha_{1}=n_{1}=E=\delta=0$.

\begin{tabular}{ccccccc}
\hline \multirow{n}{*}{} & $\boldsymbol{\alpha}$ & \multicolumn{2}{c}{$\boldsymbol{g}^{\prime}(\mathbf{0})$} & & \multicolumn{2}{c}{$\boldsymbol{f}^{\prime \prime}(\mathbf{0})$} \\
\cline { 3 - 4 } \cline { 6 - 6 } & & Chen et al. $[\mathbf{4 7}]$ & Present outcomes & & Chen et al. [47] & Present outcomes \\
\hline & 0.0 & -0.6149 & -0.61451 & & 0.5080 & 0.50913 \\
1 & 1.0 & -1.4870 & -1.4863 & & -0.9486 & -0.94820 \\
& 1.5 & -1.7996 & -1.7992 & & -1.9695 & -1.9696 \\
& & & & & 0.4639 \\
2 & 0.0 & -0.6677 & -0.6673 & & -1.2878 & -1.2875 \\
& 1.0 & -1.7418 & -1.7417 & & -2.5585 & -2.5580 \\
\hline
\end{tabular}

et al. [47] in a limiting sense for $Q, \alpha_{1}, n_{1}, E$ and $\delta$. Here, a good match is noted.

\section{Concluding remarks}

The influence of activation energy and a space dependent internal heat source in the 3D flow of nanomaterials containing gyrotactic microorganisms is examined. Comparison is provided. The main points of analysis are mentioned below:

- Both radial and tangential velocities decay from an increase in power-law index $n$, whereas a contrary trend is seen for $\alpha$;

- The temperature of nanoliquid increases for larger $N_{b}$ and $N_{t}$

- Features of $\delta$ and $\alpha_{1}$ concentration are similar;

- Concentration of nanoliquid decays for higher $E$ and $\delta$

- The behavior of $\mathrm{Pe}$ and $S_{c}$ on motile density are quite opposite;

- Skin frictions are augmented for higher $n$;

- Heat and mass transmission rates are uplifted by $N_{t}$, $N_{b}$ and $E$;

- Absolute estimations of motile microorganism transmission rate is diminished via $N_{b}$ and $E$.

\section{References}

1. Choi, S.U.S. and Eastman, J.A. "Enhancing thermal conductivity of fluids with nanoparticles", The Proceedings of the 1995 ASME International Mechanical Engineering Congress and Exposition, San Francisco, USA, ASME, FED 231/MD, 66, pp. 99-105 (1995).

2. Buongiorno, J. "Convective transport in nanofluids", J. Heat Transfer, 128(3), pp. 240-250 (2006).

3. Sheikholeslami, M., Hayat, T., and Alsaedi, A. "Numerical simulation of nanofluid forced convection heat transfer improvement in existence of magnetic field using lattice Boltzmann method", Int. J. Heat Mass Transfer, 108, pp. 1870-1883 (2017).
4. Tiwari, R.K. and Manab K.D. "Heat transfer augmentation in a two-sided lid-driven differentially heated square cavity utilizing nanofluids", Int. J. Heat Mass Transfer, 50(9-10), pp. 2002-2018 (2007).

5. Shehzad, S.A., Hayat, T., Alsaedi, A., et al. "A useful model for solar radiation", Ener. Eco. and Environ., 1(1), pp. 30-38 (2016).

6. Sheikholeslami, M., Hayat, T., and Alsaedi, A. "On simulation of nanofluid radiation and natural convection in an enclosure with elliptical cylinders", Int. J. Heat Mass Transfer, 115, pp. 981-991 (2017).

7. Alsaedi, A., Awais, M., and Hayat, T. "Effects of heat generation/absorption on stagnation point flow of nanofluid over a surface with convective boundary conditions", Commun Nonlinear Sci. and Numer. Simul., 17(11), pp. 4210-4223 (2012).

8. Hayat, T., Ullah, I., Muhammad, T., et al. "Magnetohydrodynamic (MHD) three-dimensional flow of second grade nanofluid by a convectively heated exponentially stretching surface", J. Mol. Liq., 220, pp. 1004-1012 (2016).

9. Hayat, T., Ullah, I., Waqas, M., et al. "Flow of chemically reactive magneto Cross nanoliquid with temperature-dependent conductivity", Applied Nanoscience, 8(6), pp. 1453-1460 (2018).

10. Hsiao, K.L. "Stagnation electrical MHD nanofluid mixed convection with slip boundary on a stretching sheet", Appl. Therm. Eng., 98, pp. 850-861 (2016).

11. Lin, Y., Zheng, L., Zhang, X., et al. "MHD pseudoplastic nanofluid unsteady flow and heat transfer in a finite thin film over stretching surface with internal heat generation", Int. J. Heat Mass Transfer, 84, pp. 903-911 (2015).

12. Mustafa, M., Hayat, T., Pop, I., et al. "Stagnationpoint flow of a nanofluid towards a stretching sheet", Int. J. Heat Mass Transfer, 54(25-26), pp. 5588-5594 (2011).

13. Sheikholeslami, M., Hayat, T., and Alsaedi, A. "On simulation of nanofluid radiation and natural convection in an enclosure with elliptical cylinders", Int. J. Heat Mass Transfer, 115, pp. 981-991 (2017).

14. Hayat, T., Ullah, I., Alsaedi, A., et al. "Threedimensional mixed convection flow of Sisko nanoliquid", Int. J. Mech.l Sci., 133, pp. 273-282 (2017). 
15. Hussain, Z., Hayat, T., Alsaedi, A., et al. "Threedimensional convective flow of CNTs nanofluids with heat generation/absorption effect: A numerical study", Comp. Methods Appl. Mech. Eng., 329, pp. 40-54 (2018).

16. Hayat, T., Ullah, I., Alsaedi, A., et al. "MHD flow of Powell-Eyring nanofluid over a non-linear stretching sheet with variable thickness", Results Phys., 7, pp. 189-196 (2017).

17. Ellahi, R., Zeeshan, A., Shehzad, N., et al. "Structural impact of Kerosene- $\mathrm{Al}_{2} \mathrm{O}_{3}$ nanoliquid on MHD Poiseuille flow with variable thermal conductivity: application of cooling process", J. Mol. Liq., 264, pp. 607-615 (2018).

18. Hassan, M., Marin, M., Ellahi, R., et al. "Exploration of convective heat transfer and flow characteristics synthesis by $\mathrm{Cu}-\mathrm{Ag} /$ water hybrid-nanofluids", Heat Trans. Res., 49(18), pp. 1837-1848 (2018).

19. Rashidi, S., Akar, S., Bovand, M., et al. "Volume of fluid model to simulate the nanofluid flow and entropy generation in a single slope solar still", Renew. Energy, 115, pp. 400-410 (2018).

20. Hassan, M., Marin, M., Alsharif, A., et al. "Convective heat transfer flow of nanofluid in a porous medium over wavy surface", Phys. Lett. A, $\mathbf{3 8 2}(38)$, pp. 2749-2753 (2018).

21. Akbarzadeh, M., Rashidi, S., Karimi, N., et al. "Convection of heat and thermodynamic irreversibilities in two-phase, turbulent nanofluid flows in solar heaters by corrugated absorber plates", Adv. Powder Technol., 29(9), pp. 2243-2254 (2018).

22. Alamri, S.Z., Ellahi, R., Shehzad, N., et al. "Convective radiative plane Poiseuille flow of nanofluid through porous medium with slip: an application of Stefan blowing", J. Mol. Liq., 273, pp. 292-304 (2019).

23. Shehzad, N., Zeeshan, A., Ellahi, R., et al. "Modelling study on internal energy loss due to entropy generation for non-Darcy poiseuille flow of silver-water nanofluid: an application of purification", Entropy, 20(11), p. 851 (2018).

24. Ellahi, R., Zeeshan, A., Hussain, F., et al. "Peristaltic blood flow of couple stress fluid suspended with nanoparticles under the influence of chemical reaction and activation energy", Symmetry, 11(2), p. 276 (2019).

25. Ashlin, T.S. and Mahanthesh, B. "Exact solution of non-coaxial rotating and non-linear convective flow of $\mathrm{Cu}-\mathrm{Al}_{2} \mathrm{O}_{3}-\mathrm{H}_{2} \mathrm{O}$ hybrid nanofluids over an infinite vertical plate subjected to heat source and radiative heat", J. Nanofluids, 8(4), pp. 781-794 (2019).

26. Shruthy, M., and Mahanthesh, B. "Rayleigh-Bénard convection in Casson and hybrid nanofluids: An analytical investigation", J. Nanofluids, 8(1), pp. 222-229 (2019).

27. Amala, S. and Mahanthesh, B. "Hybrid nanofluid flow over a vertical rotating plate in the presence of hall current, nonlinear convection and heat absorption", $J$. Nanofluids, 7(6), pp. 1138-1148 (2018).
28. Animasaun, I.L., Koriko, O.K., Adegbie, K.S., et al. "Comparative analysis between $36 \mathrm{~nm}$ and $47 \mathrm{~nm}$ alumina-water nanofluid flows in the presence of Hall effect", J. Therm. Anal. Calorim., 135(2), pp. 873-886 (2019).

29. Golshokooh, S., Ramazani, S.A., and Hekmatzadeh, M. "Investigating the effect of hybrid silica nanoparticles-copolymer on increasing oil recovery in a three dimensional porous media", Sci. Iran., 24(6), pp. 3466-3475 (2017).

30. AfzaliTabar, M., Alaeib, M., Khojasteh, R.R., et al. "Preference of nanoporous graphene to SingleWalled Carbon Nanotube (SWCNT) for preparing silica nanohybrid Pickering emulsion for potential Chemical Enhanced Oil Recovery (C-EOR)", Sci. Iran., 24, pp. 3491-9 (2017).

31. Karbasi, S., Zarei, M., and Foroughi, M.R. "Effects of Multi-Wall carbon Nano-Tubes (MWNTs) on structural and mechanical properties of electrospun poly (3-hydroxybutyrate) scaffold for tissue engineering applications", Sci. Iran., 23(6), p. 3145 (2016).

32. Hakeem, A.A., Ganesh, N.V., and Ganga, B. "Heat transfer of non-Darcy MHD flow of nanofluid over a stretching/shrinking surface in a thermally stratified medium with second order slip model", Sci. Iran., 22(6), p. 2766 (2015).

33. Harifi, T. and Montazer, M. " $\mathrm{TiO}_{2}$ hematite or magnetite/Ag nanoparticles synthesized on polyester fabric at various temperatures producing different superparamagnetic, self-cleaning and antibacterial textiles", Sci. Iran., 21(6), p. 2490 (2014).

34. Hayat, T., Muhammad, K., Ullah, I., et al. "Rotating squeezed flow with carbon nanotubes and melting heat", Phys. Scr., 94, p. 035702 (2019).

35. Alsedi, F.E., Ullah, I., Hayat, T., et al. "Entropy generation in non-linear mixed convective flow of nanofluid in porous space influenced by Arrhenius activation energy and thermal radiation", $J$. Therm. Anal. Calorim., 140, pp. 799-809 (2020) https://doi.org/10.1007/s10973-019-08648-0.

36. Zhou, C. and $\mathrm{Ma}, \mathrm{H}$. "Ultrasonic degradation of polysaccharide from a red algae (Porphyra yezoensis)", J. Agric. Food Chem., 54(6), pp. 2223-2228 (2006).

37. Bestman, A.R. "Natural convection boundary layer with suction and mass transfer in a porous medium", Int. J. Energ. Res., 14(4), pp. 389-396 (1990).

38. Awad, F.G., Motsa, S., and Khumalo, M. "Heat and mass transfer in unsteady rotating fluid flow with binary chemical reaction and activation energy", PloS one, 9(9), p. e107622 (2014).

39. Makinde, O.D., Olanrewaju, P.O., and Charles, W.M. "Unsteady convection with chemical reaction and radiative heat transfer past a flat porous plate moving through a binary mixture", Afrika Mat., 22(1), pp. 65-78 (2011).

40. Maleque, K. "Effects of exothermic/endothermic chemical reactions with Arrhenius activation energy 
on MHD free convection and mass transfer flow in presence of thermal radiation", J. Thermodyn., 2013 (2013).

41. Mustafa, M., Khan, J.A., Hayat, T., et al. "Buoyancy effects on the MHD nanofluid flow past a vertical surface with chemical reaction and activation energy", Int. J. Heat Mass Transfer, 108, pp. 1340-1346 (2017).

42. Abbas, Z., Sheikh, M., and Motsa, S.S. "Numerical solution of binary chemical reaction on stagnation point flow of Casson fluid over a stretching/shrinking sheet with thermal radiation", Energy, 95, pp. 12-20 (2016).

43. Zeeshan, A., Shehzad, N., and Ellahi, R. "Analysis of activation energy in Couette-Poiseuille flow of nanofluid in the presence of chemical reaction and convective boundary conditions", Results Phys., 8, pp. 502-512 (2018).

44. Hayat, T., Ullah, I., Farooq, M., et al. "Analysis of non-linear radiative stagnation point flow of Carreau fluid with homogeneous-heterogeneous reactions", $M i$ crosyst. Technol., 25(4), pp. 1243-1250 (2019).

45. Hayat, T., Ullah, I., Alsaedi, A., et al. "Numerical simulation for homogeneous-heterogeneous reactions in flow of Sisko fluid", J. Braz. Soc. Mech. Sci. Eng., 40(2), p. 73 (2018).

46. Archana, M. and Mahanthesh, B. "Exploration of activation energy and binary chemical reaction effects on nano Casson fluid flow with thermal and exponential space-based heat source", Multidiscip., 15(1), pp. 227245 (2019).

47. Chen, H., Chen, J., Geng, Y., et al. "Threedimensional boundary layer flow over a rotating disk with power-law stretching in a nanofluid containing gyrotactic microorganisms", Heat Tran. Asian Res., 47(3), pp. 569-582 (2017).

48. Zia, Q.Z., Ullah, I., Waqas, M., et al. "Cross diffusion and exponential space dependent heat source impacts in radiated three-dimensional (3D) flow of Casson fluid by heated surface", Results Phys., 8, pp. 1275-1282 (2018).

49. Hayat, T., Ullah, I., Waqas, M., et al. "Attributes of activation energy and exponential based heat source in flow of Carreau fluid with cross-diffusion effects", $J$. Non-Equil. Thermody., 44(2), pp. 203-213 (2019).
50. Khan, A.A., Usman, H., Vafai, K., et al. "Study of peristaltic flow of magnetohydrodynamic Walter's B fluid with slip and heat transfer", Sci. Iran., 23(6) pp. 2650-2662 (2016).

51. El-Aziz, M.A. and Salem, A.M. "MHD-mixed convection and mass transfer from a vertical stretching sheet with diffusion of chemically reactive species and spaceor temperature-dependent heat source", Can. J. Phys., 85(4), pp. 359-373 (2007).

52. Animasaun, I.L. "Effects of thermophoresis, variable viscosity and thermal conductivity on free convective heat and mass transfer of non-darcian MHD dissipative Casson fluid flow with suction and nth order of chemical reaction", J. Nigerian Math., 34(1), pp. 1131 (2015).

\section{Biographies}

Tasawar Hayat is a Pakistani Mathematician who has made pioneering research contributions to the area of mathematical fluid mechanics. He is considered one of the leading mathematicians working in Pakistan and, currently, is Professor of Mathematics at the QuaidiAzam University.

Ikram Ullah is a $\mathrm{PhD}$ student of Mathematics at Quaid-i-Azam University, Pakistan. He received his MS and Mphil degrees from Quaid-i-Azam University. His research interests include nanomaterials, hybrid nanomaterials, entropy analysis and heat mass transfer.

Ahmad Alsaedi is Professor in the Department of Mathematics at King Abdulaziz University, Jeddah, Saudi Arabia. He is a member of the Nonlinear Analysis and Applied Mathematics (NAAM) research group. His areas of interest include fluid dynamics, nonlinear flow analysis and flow problems in nanosystems.

Saleem Asghar is an eminent Professor at Comsats Institute of Information Technology. His research interests are fluid dynamics, wave propagation and engineering. 